\title{
MR diagnosis of vertebral artery dissection: value of 3D time-of-flight and true fast imaging with steady-state precession fusion imaging
}

\author{
Masafumi Kidoh • Takeshi Nakaura • \\ Hiroki Takashima • Makoto Yoshikawa • \\ Shouzaburou Uemura • Kazunori Harada • \\ Toshinori Hirai • Yasuyuki Yamashita
}

Received: 17 August 2012 /Revised: 5 November 2012 / Accepted: 7 November 2012 / Published online: 2 December 2012

(C) The Author(s) 2012. This article is published with open access at Springerlink.com

\begin{abstract}
Objectives We hypothesized that 3D time-of-flight (TOF) and true fast imaging with steady-state precession (trueFISP) fusion imaging could provide more information regarding the arterial vessel wall. The purpose of this study was to compare the accuracy of lesion detection and the diagnostic confidence of VAD between TOF images alone and fused TOF and true-FISP images.

Methods Fifty patients were studied: 17 had VAD and 33 had vertebral artery hypoplasia. Fusion images of the vertebral artery were reconstructed using a workstation. A receiveroperating characteristic (ROC) analysis was conducted with a continuous rating scale from 1 to 100 to compare observer performance in VAD detection. Five radiologists participated in the observer performance test, and their performances with TOF images were compared with those using fused images. Result The observers found that the mean areas under the best-fit ROC curve for TOF images alone and fused TOF images were $0.66 \pm 0.05$ and $0.93 \pm 0.04$, which were significantly different $(P<0.01)$.
\end{abstract}

\footnotetext{
M. Kidoh $(\bowtie) \cdot$ T. Nakaura

Diagnostic Radiology, Amakusa Medical Center, kameba 854-1, Amakusa, Kumamoto 863-0046, Japan

e-mail: masafkidoh@yahoo.co.jp

M. Kidoh $\cdot$ T. Nakaura $\cdot$ T. Hirai $\cdot$ Y. Yamashita

Department of Diagnostic Radiology, Graduate School of Medical

Sciences, Kumamoto University, Kumamoto, Japan

H. Takashima $\cdot$ M. Yoshikawa $\cdot$ S. Uemura

Department of Neurosurgery, Amakusa Medical Center, Amakusa, Japan

K. Harada

Department of Surgery, Amakusa Medical Center, Amakusa, Japan
}

Conclusion The fusion images provided more information regarding the arterial vessel wall. Fused images aided distinction between vertebral artery dissection versus vertebral artery hypoplasia.

Key Points

- New MR techniques can help to differentiate flowing blood from static blood products.

- Fused TOF and true-FISP images differentiate the lumen and the arterial wall, improving diagnostic performance.

- Fused images may be superior to time-of-flight MR angiography alone.

Keywords Fusion image · Vertebral artery dissection . MR angiography · True FISP · Magnetic resonance imaging

\section{Introduction}

Vertebral artery dissection is a potential cause of posterior circulation ischemia that requires high-spatial-resolution imaging for the definitive diagnosis [1]. Digital subtraction angiography (DSA) remains the gold standard for assessment of the vertebrobasilar arteries, with excellent spatial and temporal resolution [2, 3]. However, risks associated with conventional angiography include vascular injury, intracerebral complications, contrast medium nephrotoxicity and exposure to ionizing radiation. Therefore, non-invasive diagnostic techniques such as CT angiography (CTA) and magnetic resonance imaging (MRI) with MR angiography (MRA) are typically used. CTA has been shown to have high sensitivity and specificity for the diagnosis of vertebral artery dissection [4]. However, the accuracy of $\mathrm{CT}$ in the evaluation of acute ischaemic lesions in the posterior cranial fossa remains limited [5]. 
Three-dimensional (3D) time-of-flight (TOF) MRA is important as a non-invasive examination to diagnose blood flow in the brain. TOF MRA mainly reflects the blood flow within the artery, representing the inner contour of the artery. Intramural dissecting haematoma shows a typical evolution of signal intensity related to the paramagnetic effects of the products of hemoglobin breakdown and is frequently isointense to surrounding structures [6-8]. Therefore, the diagnostic performance of TOF in the detection of vertebral artery dissection is poor.

True fast MR imaging with steady-state precession (trueFISP) is a coherent steady-state technique that uses a fully balanced gradient waveform to recycle transverse magnetization. True FISP is extremely rapid relative to black-blood T1WI and T2*WI and fat-suppressed T1WI. True FISP offers a high signal-to-noise ratio and imaging efficiency; true FISP provides reliable examination of blood vessels without the need for intravenous administration of contrast medium [9]. However, true FISP is not suitable for the imaging of fast flow velocity because the reduction of signal intensity on true FISP is remarkable with a flow velocity of $50-100 \mathrm{~cm} / \mathrm{s}$, which corresponds to arterial flow velocity [10]. Therefore, true FISP is not suitable for visualizing the inner contour of the artery but can provide information regarding the outer surface appearance of the intracranial arterial wall.

There is still no consensus regarding the optimal method for diagnosing the dissection of intracranial arteries with MRI [11]. Previous reports suggested that the criteria of increase in the external diameter of the artery and narrowing of the lumen offer high diagnostic accuracy $[12,13]$. Additionally, previous reports have suggested the usefulness of fused images to depict the different anatomical information at one view [14-16]. Therefore, the combination of trueFISP and TOF MRA may be useful for more accurate diagnosis in patients with conditions such as vertebral artery hypoplasia or vertebral artery dissection. We hypothesized that the TOF and true-FISP fusion imaging could provide more information regarding the arterial vessel wall.

The purpose of this study was to compare the accuracy of lesion detection and the diagnostic confidence of vertebral artery dissection between TOF images alone and fused TOF and true-FISP images.

\section{Methods}

Patients

This prospective study was performed with institutional review board approval. Details of the study were explained, and written informed consent was obtained from all patients before the MRI examinations, including possible anonymous use of data for research purposes.
Clinical MRI examinations were performed between April 2009 and April 2012. Our study included 50 patients, 30 males and 20 females, ranging in age from 41 to 88 years (mean 68.1 years) (Table 1). Seventeen patients with vertebral artery dissection were included in the study. Thirtythree consecutive asymptomatic patients who were diagnosed with vertebral artery hypoplasia from 1 July to 17 September 2010 were also assigned to a control group.

\section{Diagnosis}

The final diagnosis of vertebral artery dissection was based on (1) compatible clinical signs and symptoms of vertebral artery dissection with definite angiographic, CTA or MRA findings of dissection in the vertebral artery; (2) no evidence of luminal irregularities, stenosis or occlusions in vertebral arteries or the other cervical and intracranial arteries that would be suggestive of atherosclerosis.

Compatible clinical signs and symptoms of vertebral artery dissection included sudden-onset occipital headache, neck pain or brain stem ischaemic symptoms [17]. The imaging findings for diagnosing vertebral artery dissection were as follows [18]: (1) a narrowed centric or eccentric lumen surrounded by crescent-shaped, mural thickening and an associated increase in external diameter; (2) an abrupt or tapered occlusive lumen and an associated increase in external diameter; (3) an aneurysmally dilated lumen or an alternatively dilated and narrowed lumen with or without crescent-shaped mural thickening or an intimal flap; and (4) healing or progression of the initial lumen abnormality on follow-up. Increased external diameter was evaluated by comparing it with the segment proximal to the dissection.

In addition to MRI examinations, conventional angiography and CTA were also performed in some patients (Table 2).

Thirty-three patients with vertebral artery hypoplasia who were asymptomatic were reviewed as a control group. Vertebral artery hypoplasia was defined as a lumen diameter of $\leq 2 \mathrm{~mm}[19]$.

Imaging

We obtained 3D TOF images and true-FISP images using 1.5-T MRI (Magnetom Avanto; Siemens Medical Systems,

Table 1 Patient characteristics

\begin{tabular}{lll}
\hline & $\begin{array}{l}\text { Vertebral artery } \\
\text { dissection }\end{array}$ & $\begin{array}{l}\text { Vertebral artery } \\
\text { hypoplasia }\end{array}$ \\
\hline $\begin{array}{l}\text { Number of } \\
\text { patients }\end{array}$ & 17 & 33 \\
$\begin{array}{l}\text { Male:female ratio } \\
\text { Age (years) }\end{array}$ & $12: 5$ & $18: 15$ \\
\hline
\end{tabular}


Table 2 Diagnostic modalities used for the diagnosis of vertebral artery dissection

\begin{tabular}{ll}
\hline & Number of patients \\
\hline Conventional angiography & $1 / 17$ \\
CTA & $5 / 17$ \\
Contrast-enhanced MRI & $7 / 17$ \\
Follow-up non-contrast-enhanced MRI & $17 / 17$ \\
\hline
\end{tabular}

Germany). The imaging parameters were as follows: 3D TOF: $18 / 2.84$ (TR/TE); flip angle, $20^{\circ}$; section thickness, $0.77 \mathrm{~mm}$; readout bandwidth, $300 \mathrm{~Hz} /$ pixel; rectangular field of view, $210 \mathrm{~mm}$; matrix, $256 \times 256$; unenhanced true FISP: $6.82 / 3.41$ (TR/TE); flip angle, $70^{\circ}$; section thickness, $0.7 \mathrm{~mm}$; readout bandwidth, $501 \mathrm{~Hz} /$ pixel; rectangular field of view, $210 \mathrm{~mm}$; matrix, $256 \times 256$. We reconstructed fused 3D TOF and true-FISP images of the vertebral artery for qualitative image analysis using a 3D-imaging workstation (Synapse Vincent, Fuji Film Medicals, Japan). Fusion images were presented using a linear rainbow color scale with seven colors ranging from purple to red. Images were displayed and saved as bitmaps.

\section{Study design}

Five radiologists participated in the observer performance test, and their performances with TOF images were compared with their performances using TOF and fused TOF and true-FISP images. The observers were three board-certified radiologists with 9-19 years of experience (mean, 12.7 years) and two radiology residents with 5-6 years of experience with brain MRI (mean, 5.5 years). All observers read brain MRI images regularly. Radiologists were blinded to the patients' identities, clinical histories and MR sequence parameters. The reading time was not limited. The observers first analysed only TOF images and next analysed two separate sets of TOF and fused TOF and true-FISP images. The images were presented in random order in each case. Radiologists were asked whether the image showed vertebral artery hypoplasia or vertebral artery dissection, and then they marked their confidence level regarding the likelihood of vertebral artery dissection by using a continuous rating scale [20]. They were asked to try to use the rating scale consistently and uniformly. Specific criteria for vertebral artery hypoplasia or vertebral artery dissection were a large mismatch between the region of the TOF and the region of the true FISP because previous reports suggested that the criteria of increase in the external diameter of the artery and narrowing of the lumen offer high diagnostic accuracy $[12,13]$. All cases were reviewed in a random order on an LCD monitor with a spatial resolution of 1,600 $\times 1,200$ (RadiForce R22, Nanao) using our PACS (Synapse, Fuji Film Medicals, Japan).
Quantitative image analysis

We calculated the differences between lumen signal (TOF) and vessel size (true FISP). Non-tortuous portions of the hypoplastic vertebral artery were measured on transverse images. Measurement was also performed at the most enlarged portion of the dissected artery. At each portion, three independent measurements were averaged. We calculated the differences between TOF and true FISP of vertebral artery hypoplasia and vertebral artery dissection using the following formula: vessel size - lumen signal.

\section{Statistical analysis}

The decision confidence ratings were analysed using ROC techniques. The data were analysed using DBM-MRMC, version 2.2, software (C.E. Metz, The University of Chicago, Chicago, IL, USA) [21-27]. To find significant differences among the methods and readers (multireadermultimodality ROC analysis), the jackknife method was applied. To compare the differences between TOF and true FISP among the two protocols, we used two-tailed Student's $t$-test. A difference with $P<0.05$ was considered significant.

\section{Results}

There was no clinically problematic misregistration between TOF and true-FISP fusion images. For the five observers, the mean area under the best-fit ROC curve $(\mathrm{Az})$ values for TOF images alone and TOF and fused TOF and true-FISP images were $0.66 \pm 0.05$ (SD) and $0.93 \pm 0.04$, respectively (Fig. 1 and Table 3 ). The difference was significant $(P<0.01)$.

The differences between TOF (lumen signal) and true FISP (vessel size) of vertebral artery dissection were significantly larger than those of the hypoplastic side of vertebral artery $(P<0.01)$ (Table 4$)$.

Representative cases with vertebral CIS artery dissection and vertebral artery hypoplasia are shown in Figs. 2 and 3.

In addition to the TOF-MRA and true-FISP imaging, T1weighted, T2-weighted, T2-star-weighted, fluid-attenuated inversion recovery and diffusion-weighted imaging were performed in all 50 patients. Ten patients with vertebral artery dissection underwent MR imaging with the additional sequences (Table 5).

\section{Discussion}

Fused images enable clear visualization of the vertebral artery, and the relationship of the inner and outer contours 


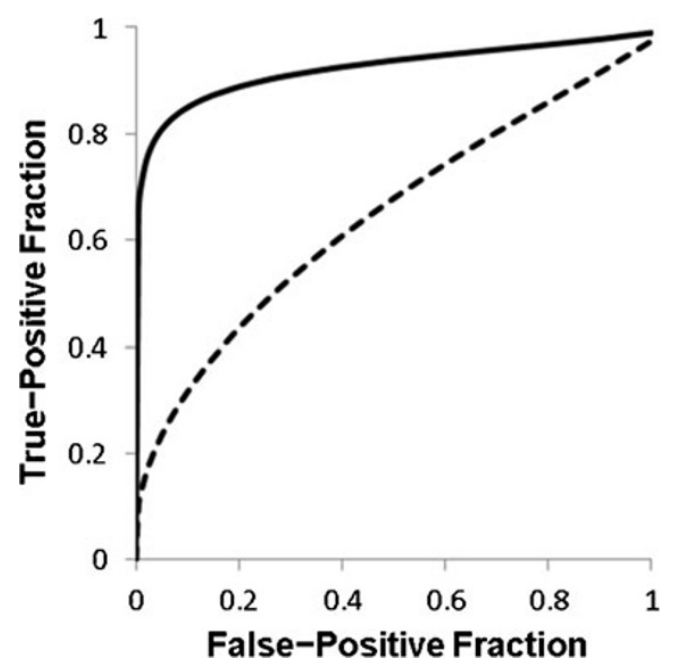

Fig. 1 Mean ROC curves for all observers who detected vertebral artery dissection. Mean area under the ROC curve increased from 0.66 \pm 0.05 (TOF images, dashed line) to $0.93 \pm 0.04$ (fused images, solid line); this difference was statistically significant $(P<0.01)$

of vessels can be assessed precisely. Fused images aided distinction between vertebral artery dissection versus vertebral artery hypoplasia. Fused images may have a supplementary role to TOF MRA images in the diagnosis of vertebral artery dissection.

Quantitative image analysis revealed that the differences between lumen signal and vessel size of vertebral artery dissection were significantly larger than those of hypoplastic side of vertebral artery. Dissections of the vertebral arteries are caused by a primary intramural haematoma or by penetration of blood into the arterial wall through a primary intimal tear [28, 29]. Blood usually enters the media at the site of intimal injury, and the dissection usually extends cranially in the same direction as the bloodstream [30-32]. The intramural haematoma usually compresses the true lumen of the artery and causes enlargement of the external diameter of the artery.

Table 3 Area under receiver-operating characteristic curve values for diagnosis of vertebral artery dissection on TOF and fused images

\begin{tabular}{lll}
\hline Observer no. & $\begin{array}{l}\text { TOF images } \\
\text { AUC }\end{array}$ & $\begin{array}{l}\text { Fused images } \\
\text { AUC }\end{array}$ \\
\hline 1 & 0.73 & 0.99 \\
2 & 0.75 & 0.95 \\
3 & 0.61 & 0.78 \\
4 & 0.64 & 0.97 \\
5 & 0.58 & 0.93 \\
Mean (SD) & $0.66(0.05)$ & $0.93(0.04)$ \\
\hline
\end{tabular}

There is no consensus regarding the optimal method for diagnosing the dissection of vertebral arteries. The diagnosis of dissection at the site of vertebral artery lesions is based on the clinical history and the findings of conventional angiography, CT or MRI. On T2weighted MRI, evidence of intra-arterial lesions, such as mural haematoma, intimal flap and plaque, are characteristic findings of vertebral artery dissection. On three-dimensional contrast-enhanced images, the double lumen at the site of dissection is visualized [33]. On T1-weighted and fluid-attenuated inversion recovery MRI images, intraluminal vertebral artery haematomas can be detected, and the pearl-and-string and the double lumen signs are characteristic MRA findings of vertebral artery dissection [33-36].

The diagnostic performance of TOF in the detection of vertebral artery dissection is poor. TOF MRA is currently the most commonly used pulse sequence in the MR evaluation of intracranial arteries. However, the sensitivity of TOF MRA for the detection of vertebral artery dissection is $60 \%[12,37]$. In comparison, multidetector 3D-CTA has good accuracy for the diagnosis of vertebral artery dissection, with sensitivity and specificity of $100 \%$ and $98 \%$, respectively. If the vessel dissection enlarges distally, the MR signals in the pseudo-lumen of dissected vessels are affected by the turbulence of blood flow, and TOF MRA may not clearly depict blood flow within the pseudo-lumen [38-40]. Thus, TOF MRA may underestimate the area of dissection.

True FISP is not suitable for visualizing the inner contour of the artery but is suitable for visualizing its outer contour. The true-FISP technique was originally designed for improved visualization of the cerebrospinal fluid [41]. Attributes of the true-FISP sequence include a relatively high image signal-to-noise ratio and rapid data acquisition, which yields diminished sensitivity to motion. These attributes are favorable for depicting the outer surface appearance of the arterial wall. However, pulsation artifacts (inhomogeneous signal) within the arteries are frequent on true-FISP images [42]. Additionally, the signal intensity on trueFISP is reduced when the flow velocity is more than $30 \mathrm{~cm} / \mathrm{s}$ [10]. Therefore, true FISP is not suitable for the imaging of fast flow velocity; that is, true-FISP

Table 4 The differences between TOF and true FISP

\begin{tabular}{llll}
\hline & $\begin{array}{l}\text { Vertebral } \\
\text { artery } \\
\text { hypoplasia }\end{array}$ & $\begin{array}{l}\text { Vertebral } \\
\text { artery } \\
\text { dissection }\end{array}$ & $\begin{array}{l}P \\
\text { value }\end{array}$ \\
\hline $\begin{array}{c}\text { The differences between } \\
\text { TOF and true FISP }(\mathrm{mm})\end{array}$ & $0.3 \pm 0.2$ & $3.6 \pm 1.6$ & $<0.01$ \\
\hline
\end{tabular}


Fig. 2 a A 63-year-old man complaining of headache. The left vertebral artery dissection on 3D TOF $(A$ and $B)$ is not clear (arrow). Its shape is not equal to what is evident on fused images. Fused image shows enlargement of the overall vessel diameter with an intramural haematoma in the left vertebral artery. Fused image enabled precise assessment of the relationship between the inner and outer contours of the vessels (arrow). (A) 3D TOF, (B) 3D TOF (MIP), (C) TOF and true-FISP fusion (axial), $(D)$ TOF and true-FISP fusion (oblique). b Four months later, he underwent follow-up MRI. Vertebral artery dissection was confirmed on imaging followup by monitoring normalization of the vessel lumen. Follow-up MRI scan indicated normalization of the vessel lumen (arrow). (A) 3D TOF, (B) 3D TOF (MIP), $(C)$ TOF and true-FISP fusion (axial), $(D)$ TOF and true-FISP fusion (oblique) a
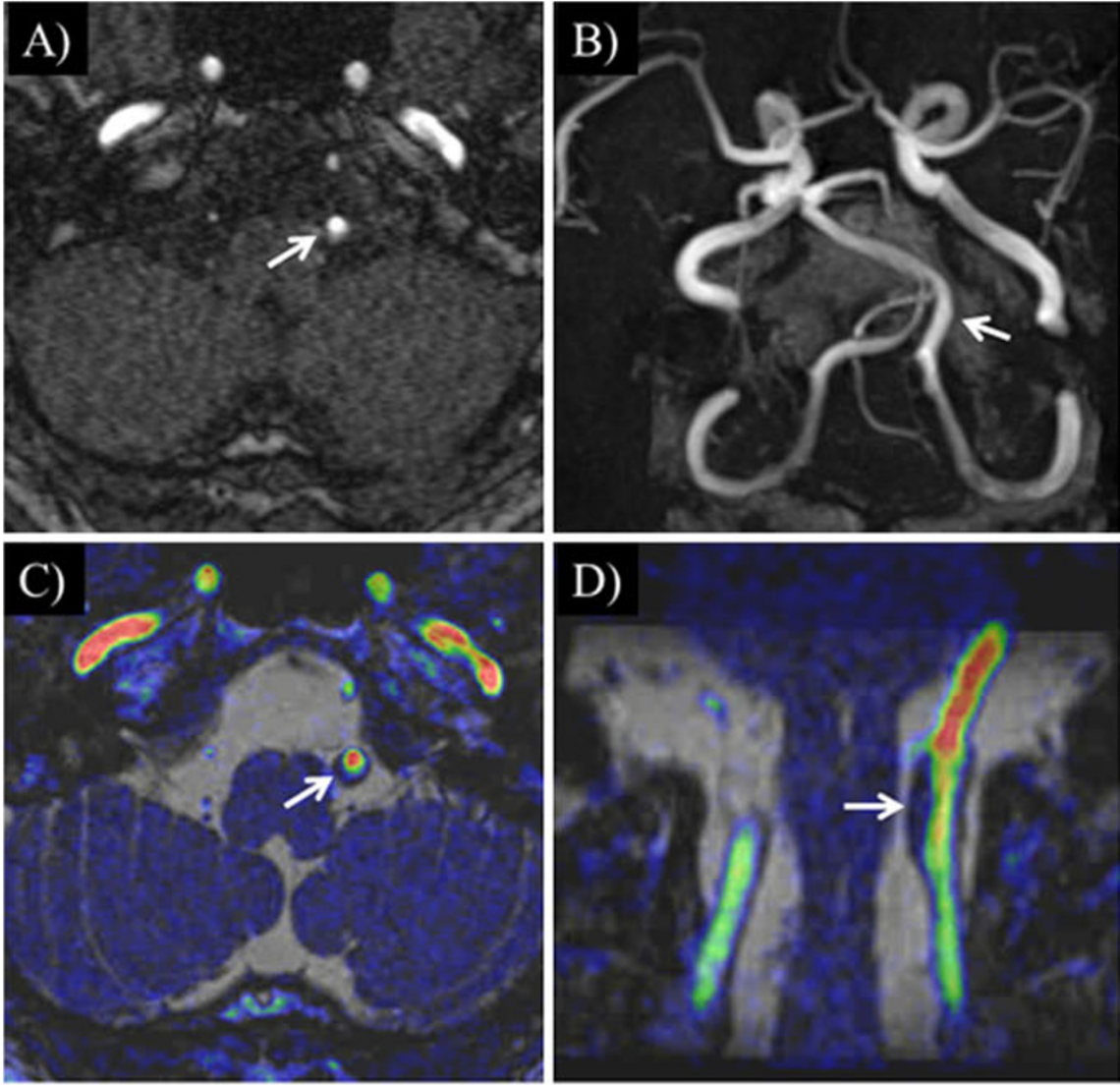

b
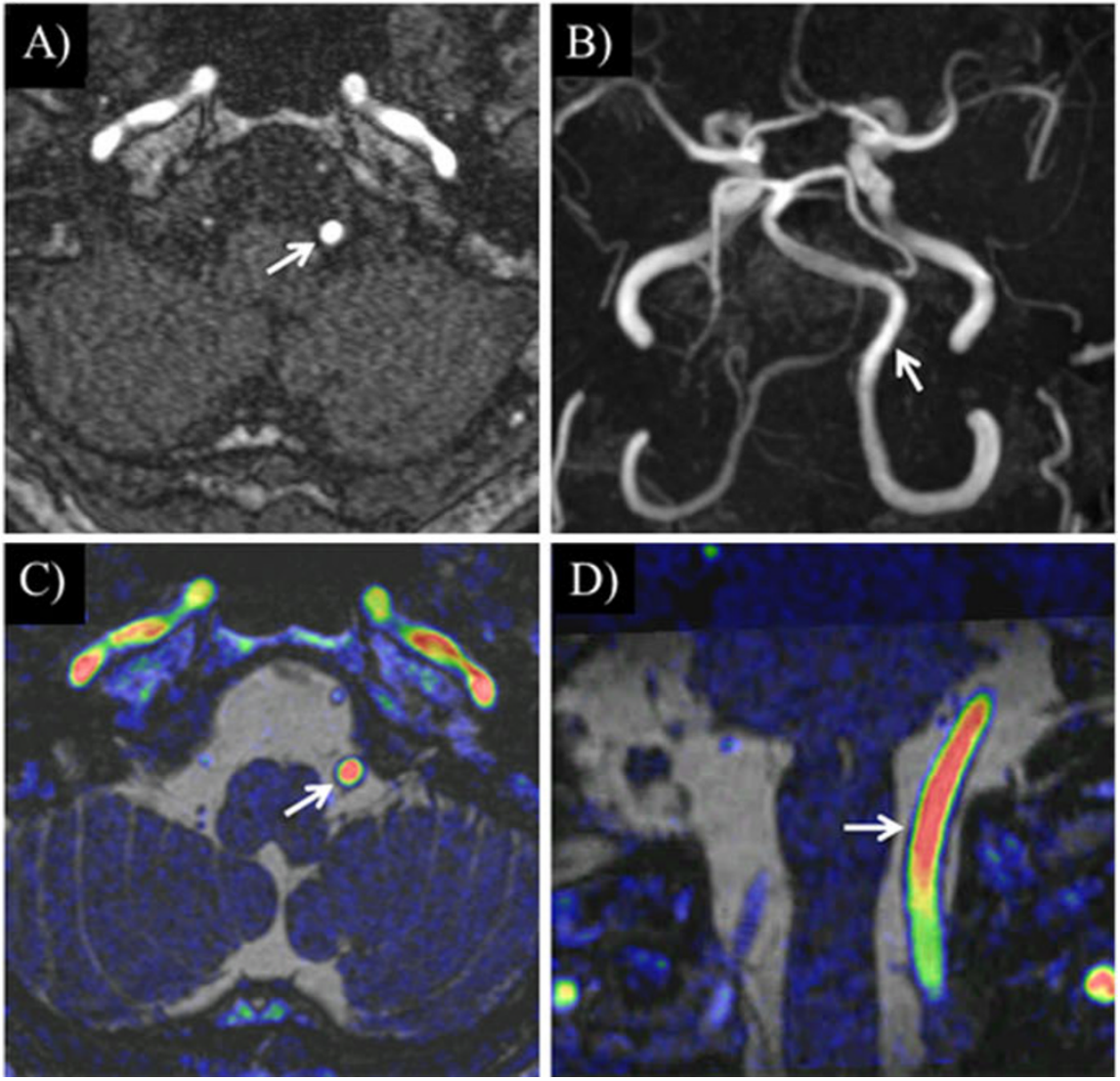
Fig. 3 A 67-year-old man. A case of left hypoplastic vertebral artery. The left vertebral artery is not seen clearly on $3 \mathrm{D}$ TOF (a and b) (arrow). A small and hypoplastic left vertebral artery is confirmed on fused imaging ( $\mathbf{c}$ and $\mathbf{d}$ ). Fused image shows no abnormalities along the course of the vertebral artery (arrow). a 3D TOF, b 3D TOF (MIP), c TOF and trueFISP fusion (axial), d TOF and true-FISP fusion (oblique)
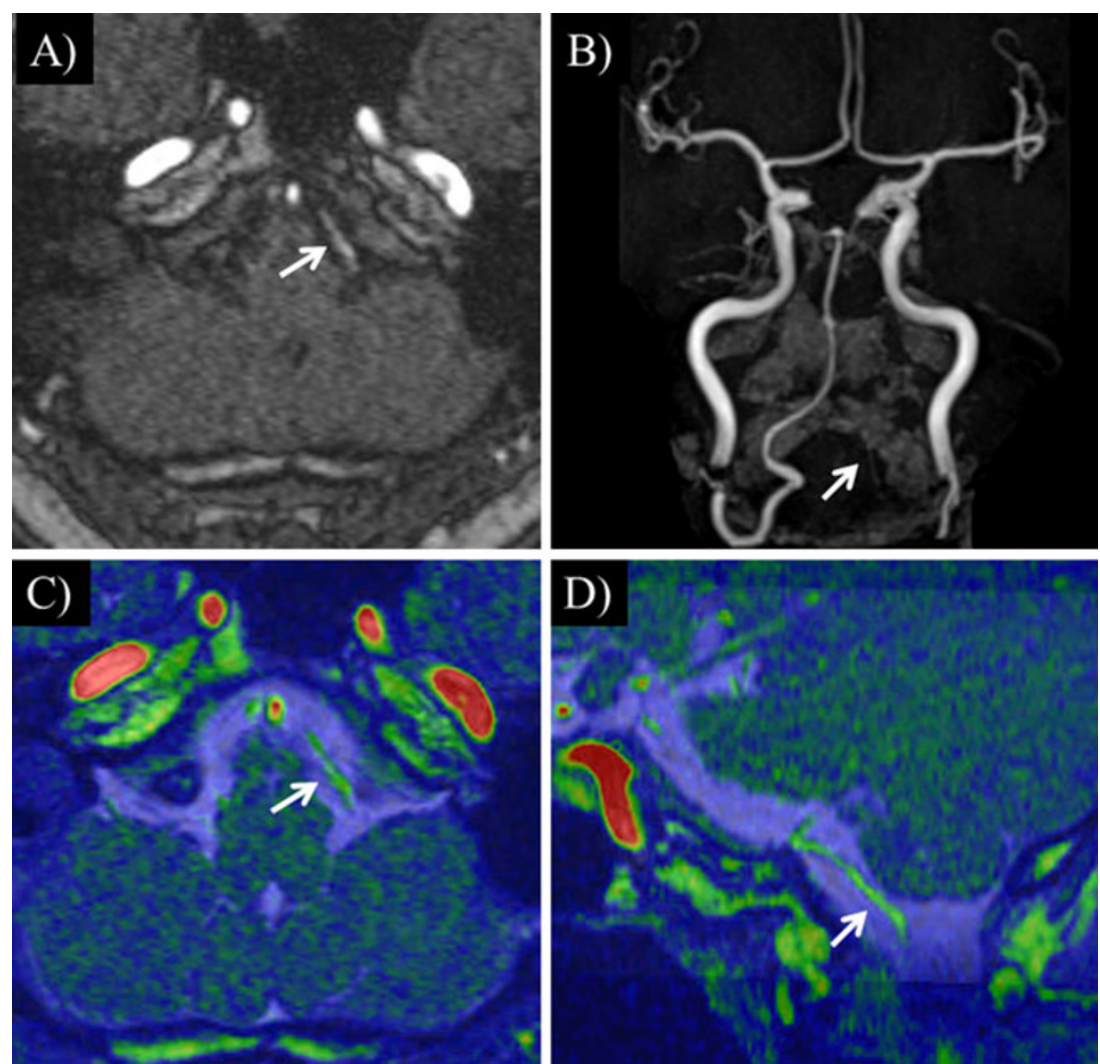

MR imaging alone is not suitable for visualizing the inner contour of the artery. True FISP reflects the outer surface appearance of the intracranial arterial wall, whereas TOF MRA mainly reflects the blood flow within the artery, representing the inner contour of the artery.

Use of fused imaging is a good diagnostic method for vertebrobasilar disease. Three-dimensional TOF MRA evaluation of the absence or decrease in the blood flow signal may not clearly discriminate vertebral artery hypoplasia from vertebral artery dissections. Therefore, we consider that evaluation of the dissection of vessels should include examination of both the inner and the outer contours of the vessels. The fused imaging reflects the outer surface appearance of the intracranial arterial wall and the inner contour of the artery, whereas TOF MRA mainly reflects the blood flow within the artery, representing the inner contour of the artery. This may be the difference from the established vascular imaging methods such as DSA, TOF MRA, CTA or black blood MRA [43-45], which disclose the vascular inner contour. The relationship between the inner and outer contours of the vessels is clearly depicted on fused images. Therefore, we think this imaging technique provides additional information in the detection of vertebral artery dissection.

Our study had limitations. The major limitations to this pilot study include the small sample and lack of a normal
Table 5 Additional sequences in the imaging of vertebral artery dissection

$V I B E$ volumetric interpolated breath-hold examination, $C E$ contrast-enhanced, FS fat saturated, TSE turbo spin echo, SPACE sampling perfection with application optimized contrasts using different flip angle evolution

\begin{tabular}{|c|c|c|c|c|c|}
\hline Patient no. & Dynamic 3D-VIBE & CE-T1WI & FS-CE-T1WI & CE-TOF-MRA & 3D TSE (SPACE) \\
\hline 1 & $\bigcirc$ & $\bigcirc$ & & & \\
\hline 2 & $\bigcirc$ & & & & \\
\hline 3 & $\bigcirc$ & & & $\bigcirc$ & \\
\hline 4 & $\bigcirc$ & & & $\bigcirc$ & $\bigcirc$ \\
\hline 5 & $\bigcirc$ & $\bigcirc$ & & $\bigcirc$ & \\
\hline 6 & & $\bigcirc$ & $\bigcirc$ & & $\bigcirc$ \\
\hline 7 & & $\bigcirc$ & $\bigcirc$ & & \\
\hline 8 & & $\bigcirc$ & & $\bigcirc$ & \\
\hline 9 & & $\bigcirc$ & & $\bigcirc$ & $\bigcirc$ \\
\hline 10 & & & & & $\bigcirc$ \\
\hline
\end{tabular}


vessel group. To confirm the coherence between TOF and true FISP, we might have to consider a control group of normal vessels. Second, complete blinding of the readers to the sequence type was not possible because their signal characteristics are quite typical. Third, TOF imaging may be affected by flow changes compared with contrast-enhanced MRA. Therefore, we cannot exclude the possibility that luminal stenosis was overestimated on fused images. Lastly, fusion imaging of TOF and true FISP only evaluates the relationship between the inner and outer contours of vessels, without differentiating between arterial wall thickening due to arteriosclerosis and dissected arteries. To exclude atherosclerotic diseases, clinical presentations, supportive radiologic evidence and follow-up imaging are needed.

In conclusion, the fusion images provided more information regarding the arterial vessel wall. Fused images aided distinction between vertebral artery dissection versus vertebral artery hypoplasia. Fused images may have a supplementary role to play in TOF MRA images in the diagnosis of vertebral artery dissection.

\section{Conflict of interest None}

\section{Financial disclosure None}

Open Access This article is distributed under the terms of the Creative Commons Attribution License which permits any use, distribution, and reproduction in any medium, provided the original author(s) and the source are credited.

\section{References}

1. Gui L, Shi GS, Li GJ et al (2010) Spontaneous vertebral artery dissection: report of 16 cases. Neurol India 58(6):869-874

2. Teasdale E, Zampakis P, Santosh C, Razvi S (2011) Multidetector computed tomography angiography: Application in vertebral artery dissection. Ann Indian Acad Neurol 14(1):35-41

3. Pugliese F, Crusco F, Cardaioli G et al (2007) CT angiography versus colour-Doppler US in acute dissection of the vertebral artery. Radiol Med 112(3):435-443

4. Vertinsky AT, Schwartz NE, Fischbein NJ, Rosenberg J, Albers GW, Zaharchuk G (2008) Comparison of multidetector CT angiography and MR imaging of cervical artery dissection. AJNR Am J Neuroradiol 29(9):1753-1760

5. Chen CJ, Tseng YC, Lee TH, Hsu HL, See LC (2004) Multisection $\mathrm{CT}$ angiography compared with catheter angiography in diagnosing vertebral artery dissection. AJNR Am J Neuroradiol 25(5):769-774

6. Goldberg HI, Grossman RI, Gomori JM, Asbury AK, Bilaniuk LT, Zimmerman RA (1986) Cervical internal carotid artery dissecting hemorrhage: diagnosis using MR. Radiology 158(1):157-161

7. Mascalchi M, Bianchi MC, Mangiafico S et al (1997) MRI and MR angiography of vertebral artery dissection. Neuroradiology 39 (5):329-340

8. Kitanaka C, Tanaka J, Kuwahara M, Teraoka A (1994) Magnetic resonance imaging study of intracranial vertebrobasilar artery dissections. Stroke 25(3):571-575
9. Spuentrup E, Buecker A, Stuber M, Gunther RW (2001) MRvenography using high resolution True-FISP. RoFo: Fortschritte auf dem Gebiete der Rontgenstrahlen und der Nuklearmedizin 173 (8):686-690

10. Uchikoshi M, Ueda T, Nishiki S et al (2003) Fundamental study of the relation between flow velocity and signal intensity in the TrueFISP sequence. Nihon Hoshasen Gijutsu Gakkai Zasshi 59 (12):1529-1534

11. Katsuno M, Kobayashi S (2011) Diagnosis of vertebral artery dissection with basiparallel anatomical scanning magnetic resonance imaging. J Nihon Med Sch 78(6):367-373

12. Levy C, Laissy JP, Raveau V et al (1994) Carotid and vertebral artery dissections: three-dimensional time-of-flight MR angiography and MR imaging versus conventional angiography. Radiology 190(1):97-103

13. Rodallec MH, Marteau V, Gerber S, Desmottes L, Zins M (2008) Craniocervical arterial dissection: spectrum of imaging findings and differential diagnosis. Radiographics: a review publication of the Radiological Society of North America, Inc 28(6):1711-1728

14. Karlo CA, Steurer-Dober I, Leonardi M, Pfirmann CW, Zanetti M, Hodler J (2010) MR/CT image fusion of the spine after spondylodesis: a feasibility study. Eur Spine J 19(10):1771-1775

15. Plouin-Gaudon I, Bossard D, Ayari-Khalfallah S, Froehlich P (2010) Fusion of MRIs and CT scans for surgical treatment of cholesteatoma of the middle ear in children. Arch Otolaryngol Head Neck Surg 136(9):878-883

16. Watanabe Y, Nakazawa T, Yamada N et al (2009) Identification of the distal dural ring with use of fusion images with 3D-MR cisternography and MR angiography: application to paraclinoid aneurysms. AJNR Am J Neuroradiol 30(4):845-850

17. Silbert PL, Mokri B, Schievink WI (1995) Headache and neck pain in spontaneous internal carotid and vertebral artery dissections. Neurology 45(8):1517-1522

18. Naggara O, Louillet F, Touze E et al (2010) Added value of highresolution MR imaging in the diagnosis of vertebral artery dissection. AJNR Am J Neuroradiol 31(9):1707-1712

19. Fisher CM GI, Okabe N et al (1965) Atherosclerosis of the carotid and vertebral arteries - extracranial and intracranial. J Neuropathol Exp Neurol 24(3):455-476

20. Metz CE, Herman BA, Shen JH (1998) Maximum likelihood estimation of receiver operating characteristic (ROC) curves from continuously-distributed data. Stat Med 17(9):1033-1053

21. Dorfman DD, Berbaum KS, Metz CE (1992) Receiver operating characteristic rating analysis. Generalization to the population of readers and patients with the jackknife method. Invest Radiol 27 (9):723-731

22. Dorfman DD, Berbaum KS, Lenth RV, Chen YF, Donaghy BA (1998) Monte-Carlo validation of a multireader method for receiver operating characteristic discrete rating data: factorial experimental design. Acad Radiol 5(9):591-602

23. Hillis SL, Berbaum KS (2004) Power estimation for the DorfmanBerbaum-Metz method. Acad Radiol 11(11):1260-1273

24. Hillis SL, Obuchowski NA, Schartz KM, Berbaum KS (2005) A comparison of the Dorfman-Berbaum-Metz and ObuchowskiRockette methods for receiver operating characteristic (ROC) data. Stat Med 24(10):1579-1607

25. Hillis SL, Berbaum KS (2005) Monte Carlo validation of the Dorfman-Berbaum-Metz method using normalized pseudovalues and less data-based model simplification. Acad Radiol 12 (12):1534-1541

26. Hillis SL (2007) A comparison of denominator degrees of freedom methods for multiple observer ROC analysis. Stat Med 26(3):596619

27. Hillis SL, Berbaum KS, Metz CE (2008) Recent developments in the Dorfman-Berbaum-Metz procedure for multireader ROC study analysis. Acad Radiol 15(5):647-661 
28. Schievink WI (2001) Spontaneous dissection of the carotid and vertebral arteries. N Engl J Med 344(12):898-906

29. Muller BT, Luther B, Hort W, Neumann-Haefelin T, Aulich A, Sandmann W (2000) Surgical treatment of 50 carotid dissections: indications and results. J Vasc Surg 31(5):980-988

30. Schievink WI, Mokri B, O'Fallon WM (1994) Recurrent spontaneous cervical-artery dissection. N Engl J Med 330(6):393-397

31. Arnold M, Bousser MG, Fahrni G et al (2006) Vertebral artery dissection: presenting findings and predictors of outcome. Stroke 37(10):2499-2503

32. de Bray JM, Baumgartner RW (2005) History of spontaneous dissection of the cervical carotid artery. Arch Neurol 62(7):1168-1170

33. Nakagawa K, Touho H, Morisako T et al (2000) Long-term followup study of unruptured vertebral artery dissection: clinical outcomes and serial angiographic findings. J Neurosurg 93(1):19-25

34. Hosoya T, Adachi M, Yamaguchi K, Haku T, Kayama T, Kato T (1999) Clinical and neuroradiological features of intracranial vertebrobasilar artery dissection. Stroke 30(5):1083-1090

35. Naito I, Iwai T, Sasaki T (2002) Management of intracranial vertebral artery dissections initially presenting without subarachnoid hemorrhage. Neurosurgery 51(4):930-937, discussion 937-938

36. Provenzale JM, Morgenlander JC, Gress D (1996) Spontaneous vertebral dissection: clinical, conventional angiographic, $\mathrm{CT}$, and MR findings. J Comput Assist Tomogr 20(2):185-193

37. Zuber M, Meary E, Meder JF, Mas JL (1994) Magnetic resonance imaging and dynamic CT scan in cervical artery dissections. Stroke 25(3):576-581
38. Jou LD, Wong G, Dispensa B et al (2005) Correlation between lumenal geometry changes and hemodynamics in fusiform intracranial aneurysms. AJNR Am J Neuroradiol 26 (9):2357-2363

39. Nederkoorn PJ, van der Graaf Y, Eikelboom BC, van der Lugt A, Bartels LW, Mali WP (2002) Time-of-flight MR angiography of carotid artery stenosis: does a flow void represent severe stenosis? AJNR Am J Neuroradiol 23(10):1779-1784

40. Mustert BR, Williams DM, Prince MR (1998) In vitro model of arterial stenosis: correlation of MR signal dephasing and transstenotic pressure gradients. Magn Reson Imaging 16(3):301-310

41. Haacke EM, Wielopolski PA, Tkach JA, Modic MT (1990) Steady-state free precession imaging in the presence of motion: application for improved visualization of the cerebrospinal fluid. Radiology 175(2):545-552

42. Carr JC, Simonetti O, Bundy J, Li D, Pereles S, Finn JP (2001) Cine MR angiography of the heart with segmented true fast imaging with steady-state precession. Radiology 219(3):828-834

43. Edelman RR, Mattle HP, Wallner B et al (1990) Extracranial carotid arteries: evaluation with "black blood" MR angiography. Radiology 177(1):45-50

44. Bosmans H, Wilms G, Marchal G, Demaerel P, Baert AL (1995) Characterisation of intracranial aneurysms with MR angiography. Neuroradiology 37(4):262-266

45. Naganawa S, Ito T, Shimada $\mathrm{H}$ et al (1997) Cerebral black blood MR angiography with the interleaved multi-slab three-dimensional fast spin echo sequence. Radiat Med 15(6):385-388 